[4] McKeon PO, Hertel J, Bramble D, Davis I. The foot core system: a new paradigm for understanding intrinsic foot muscle function. Br J Sports Med. 2015;49(5):290.

Disclosure of Interest: None declared

DOI: 10.1136/annrheumdis-2018-eular.4503

\section{AB1438-HPR THE ASSESSMENT OF IMAGERY ABILITY IN PATIENTS WITH FAMILIAL MEDITERRANEAN FEVER}

A. Alikaj', A. Büyükaslan ${ }^{1}, \underline{\text { S Uğurlu }}{ }^{2}$, B. Dilek ${ }^{1} .{ }^{1}$ Physiotherapy and Rehabilitation, Istanbul Medipol University; ${ }^{2}$ Rheumatology, Cerrahpasa Medical Faculty, Istanbul, Turkey

Background: Studies have shown that individuals with FMF are more restricted in terms of physical function than the normal population and that depression and anxiety are more common in these individuals. Catastrophizing is the strongest psychological factor associated with pain. Imagery is a cognitive process fundamental to motor learning and performance. It is also a mental technique that can be utilised in many ways. A main function of imagery is to aid self-regulation of thoughts, feelings, and behaviours. Studies have shown to be more effective for individuals displaying a higher level of imagery ability when using imagery to improve motor and motivational outcomes, including self-efficacy. Several studies suggest that pain-related imagery may help to reduce distress and increase behavioural flexibility in individuals suffering from chronic pain. However, there is no published imagery research in FMF patients.

Objectives: The aim of this study was to assess imagery ability and pain catastrophizing in patients with familial mediterranean fever.

Methods: Between October and December 2017, 30 participants diagnosed with FMF were recruited through the Division of Rheumatology Department of Internal Medicine Cerrahpasa Medical Faculty University of Istanbul. The Istanbul Medipol University Ethics Committee approved the study. Demographic and participant characteristic information were recorded. Clinical data collected were: Age onset of FMF, age of diagnosis were inquired. Pain catastrophizing was assessed with Pain Catastrophizing Scale (PCS) and imagery ability was assessed with Movement Imagery Questionnaire- 3 (MIQ-3).A total PCS score of 30 represents clinically relevant level of catastrophizing. MIQ-3 is a 12-item questionnaire to assess individuals ability to image four basic movements: a knee lift, jump, arm movement, and waist bend. Ease of imaging is measured in both visual and kinesthetic modalities. For each item, participants read a description of the movement. Then, they physically perform the movement before assuming the same starting position to either visually or kinesthetically image the movement. Following this step, participants rate their ease of imaging on a 7-point Likert-type scale ranging from 1 to 7 (very hard/easy to see/feel). After the items for each subscale are averaged, a higher score represents a greater ease of imaging.

Abstract AB1438HPR - Table 1. Demographic and clinical characteristics of study population

\begin{tabular}{|l|l|}
\hline Variables & Mean $\pm S D$ \\
& Median (min-max) \\
\hline Age & $32.37 \pm 11(18-56)$ \\
\hline BMI (KG/m²) & $24.39 \pm 6.1(17.3-40.3)$ \\
\hline Age of Diagnosis & $22.20 \pm 14.7(2.54)$ \\
\hline Disease Duration & $10.17 \pm 7.6(2-28)$ \\
\hline Kinesthetic Imagery Ability & $14.99 \pm 4.5(3.1-21.1)$ \\
\hline Internal Imagery Ability & $13.84 \pm 5.3(3.16-20.0)$ \\
\hline External Imagery Ability & $12.41 \pm 4.7(3.8-19.0)$ \\
\hline Pain Catastrophizing Scale & $23.27 \pm 12.5(6-44)$ \\
\hline
\end{tabular}

Results: The study included 27 female, 3 male. Mean age was $32 \pm 11$ years, mean BMl was $24 \pm 6.1 \mathrm{~kg} / \mathrm{m}^{2}$; (table 1). Kinesthetic imagery ability was higher than internal and external visual imagery. There was no significant relalationship between imagery and pain catastrophizing severity.

Conclusions: According to previous studies people with chronic pain had pain-related imagery, catastrophizing, and distress related in proportion with each other but in our study, we didn't find any significant relationship between imagery and catastrophizing. This may be due to smal sample size or the pattern of pain in FMF which is periodic, intermittent differently from chronic pain. Each patient with rheumatic disease should be addressed as a composite biopsychosocial being with unique characteristics and needs. Previous study have shown that imagery is an effective treatment for neuropathic and chronic pain. We suggest that imagery may be an effective method for management of pain in patients with FMF.

Disclosure of Interest: None declared

DOI: 10.1136/annrheumdis-2018-eular.5669

\section{AB1439-HPR THE EFFECTS OF DIFFERENT EXERCISE PROTOCOLS ON FUNCTIONAL STATUS AND AEROBIC CAPACITY IN PATIENTS WITH ANKYLOSING SPONDYLITIS}

S Oksuz ${ }^{1}$, E. Unal ${ }^{2}$, G. Arın ${ }^{3} .{ }^{1}$ Physiotherapy and Rehabilitation, Health sciences, Nicosia, Cyprus; ${ }^{2}$ physiotherapy and rehabilitation, health science; ${ }^{3}$ Physiotherapy and Rehabilitation, Health sciences, Ankara, Turkey

Background: Although there is the emphasis on the importance of lifelong regular exercise to improve the efficacy of medication in the treatment of ankylosing spondylitis (AS) patients, there is a lack of information about the safe exercise dosage in clinical practice.

Objectives: In this study, we aimed to investigate the effects of different exercise protocols on functional status and aerobic capacity in patients with ankylosing spondylitis.

Methods: Thirty-one ankylosing spondylitis patients were evaluated and grouped according to their arrival order. Patients' spinal mobility (Bath Ankylosing Spondylitis Mobility Index), disease activity (Bath Ankylosing Spondylitis Disease Activity Index), flexibility (back scratch test), pulmonary functions (forced vital capacity with pulmonary function test, maximal inspiratory and expiratory pressures with respiratory muscle strength test), aerobic capacity (oxygen consumption test with submaximal modified Bruce protocol), fatigue level (Fatigue Severity Scale) and sleep quality (Pittsburgh Sleep Quality Index) were assessed. Group $1 \quad(n=16)$ did both aerobic training and clinical pilates exercises, while group $2(n=15)$ only did aerobic training. Patients did exercises for 8 weeks, 3 days a week under the supervision of a physiotherapist and then measurements were repeated.

Results: According to the measurements, it was found that disease activity level, respiratory muscle strength was improved $(p<0.05)$ in both groups. When clinical pilates exercise was given additionally to aerobic training spinal mobility (BASMI score), upper extremities flexibility, forced vital capacity, fatigue severity and sleep quality $(p<0.05)$ was also improved.

Conclusions: As a result of the study, it was noted that when clinical pilates exercises applied together with the aerobic exercise training in ankylosing spondylitis patients, effectiveness on functional status and aerobic capacity was increased.

Disclosure of Interest: None declared

DOI: 10.1136/annrheumdis-2018-eular.3980

\section{AB1440-HPR YOGA-THERAPY FOR RHEUMATOID ARTHRITIS: RAPID IMPROVEMENT IN PROMS}

V Sadana ${ }^{1}$, T. Cartwright ${ }^{1}$, M. Cahill ${ }^{1}$, K. Anie ${ }^{1}$, C.B. Colaço ${ }^{2} .{ }^{1} C M H, L N W H T$; ${ }^{2}$ LNWH NHS Trust, Rheumatology Unit, CMH, LNWUH NHS Trust, London, UK

Background: Rheumatoid Arthritis (RA) is associated with mood disorders and poor quality of life (QOL) Chorus et al., 2003 Yoga therapy (Y-T) has been used in several Long Term Conditions. Khalsa et al, 2016. Objectives: This study investigated: a) impact of a 16 week Y-T intervention on functional outcomes and QOL in 10 adult-onset RA patients, b) acceptability and experiences of the intervention.

Methods: Ten adult RA patients (Ages: 29-71 Y; RA duration: 115 years) consented to 10 individual $\mathrm{Y}$-T sessions (weekly $\times 4$; biweekly $\times 6)$ with a yoga therapist in a standard consulting room. The intervention was tailored to the needs and abilities of each patient and included: breath-centred physical yoga postures, breathing and visualisation techniques, mantras and meditation, and Lifestyle/behavioural strategies. All participants completed measures to assess changes in health pre- and postintervention (EQ-5D and HADS) and took part in a semi-structured 
interview in a mixed methods design. Thematic analysis was applied to interview data.

Results: $98 / 100$ sessions were attended by participants.

Abstract AB1440HPR - Table 1. Pre-Post 10 sessions Y-T metrics

\begin{tabular}{lccc}
\hline Pre-Post Y-T Change: & PRE & POST & CHANGE \\
\hline Mean Depression & 6.70 & 2.30 & $-65 \%$ \\
Mean Anxiety & 9.40 & 4.80 & $-48 \%$ \\
Mean HAQ & 1.10 & $0.85^{*}$ & $-23 \%$ \\
Mean Pain Score (HAQ) & 5.66 & 2.40 & $-58 \%$ \\
Mean Health Score & 5.10 & 1.72 & $-66.3 \%$ \\
(HAQ) & & & $+39.6 \%$ \\
Mean health Utility (EQ- & 6.01 & $8.40^{* *}$ & \\
5D) & & &
\end{tabular}

*8/10 HAQ reduced $>0.35=/>$ MID (minimal important difference);

**6/10 participants achieved EQ5D maximum score $=1$

Qualitative findings indicated that considerable value was placed on the therapeutic function of the consultation, of being listened to and receiving advice on concerns. Therapeutic mechanisms appeared to reflect tailored physical practices alongside psychosocial techniques which promoted psychological wellbeing and increased perceptions of control and self-efficacy. Several reported reductions in their medication and broader benefits such as improved sleep, mood and energy. All but one participant reported positive changes to their RA symptoms, including pain reduction, greater mobility and joint flexibility.

Conclusions: This novel pilot YT intervention was positively received by patients with RA, with high levels of adherence to both the course of treatments and the tailored home practice. The results suggest that yoga therapy has potential as an adjunct therapy to improve RA symptoms, increase self-care behaviours and address negative mood. Whilst promising, a larger multi-centre study is required to evaluate the therapeutic and cost-effectiveness of $\mathrm{Y}$-T.

\section{REFERENCES:}

[1] Chorus AM, Miedema HS, Boonen A, Van Der Linden S. (2003). Quality of life and work in patients with rheumatoid arthritis and ankylosing spondylitis of working age. Ann Rheum Dis. 62(12):1178-1184.

[2] Khalsa, SB, Cohen, L, McCall, T \& Telles, S (2016) (Eds). The Principles and Practice of Yoga in Health Care. Handspring Publishing.

Acknowledgements: LNWH Trust Fund, CMH Rheumatology Patient Support Group,University of Westminster Department of Health Psychology. Disclosure of Interest: V. Sadana Grant/research support from: Study Charity Grant NWLHT, T. Cartwright: None declared, M. Cahill: None declared, K. Anie: None declared, C. B. Colaço: None declared

DOI: 10.1136/annrheumdis-2018-eular.6276

\section{HPR Patients' perspectives, functioning and health (descriptive: qualitative or quantitative)}

\section{AB1441-HPR DOES THE INCIDENCE OF INFECTIONS INCREASE IN PATIENTS WITH CHRONIC ARTHRITIS AND OSTEOPOROSIS TREATED WITH DENOSUMAB AND BIOLOGICAL THERAPY?}

A Lopez Esteban ${ }^{1}$, F. Garcia ${ }^{2}$, I. Janta ${ }^{3}$, J.G. Ovalles ${ }^{3}$, B. Serrano ${ }^{2}$, J.C. Sanchez ${ }^{2}$ C. Garaballu ${ }^{2}$, C. Gonzalez ${ }^{2}$, T. Del Rio ${ }^{2}$, J. Lopez Longo ${ }^{2}$, C. Gonzalez ${ }^{2}$, I. Monteagudo ${ }^{2}$, J.C. Nieto $2 .{ }^{1}$ Reumatología; ${ }^{2}$ Rheumatology, Hospital Gregorio Marañón, Madrid, Spain; ${ }^{3}$ Reumatología, Hospital Gregorio Marañón, Madrid, -

Background: Biological therapies (BT) have changed the prognosis of chronic arthritis (CA); however, they have associated the increased risk of infections. Denosumab (DNS) is the first biologic approved for the treatment of osteoporosis (OP) and is also associated with an increased risk of infections.

Objectives: To evaluate the incidence of infections in patients with CA and OP treated with DNS alone or associated to another biological treatment.

Methods: A prospective observational study was designed in which all patients with OP who received treatment with DNS between January 2013 and December 2017 were included. The group of patients with CA (RA, EA, PsA) were treated, in addition to DNS as treatment for their $\mathrm{OP}$, with BT. Follow-up was carried out in the rheumatology nursing examination room every 6 months. Demographic data, disease charaacteristics, infections and associated comorbidities were collected. Serious infections were defined as those that required admission, suspension of therapy or death.

Results: 220 patients were included (81.1\% women). The main diagnoses were OP $112(51 \%)$ and AC 76 (34.5\%) [RA 58 patients (26.3\%), SpA $8(3.6 \%)$, Psa $10(4.5 \%)]$ and other rheumatologic diagnoses $32(14.5 \%)$. Demographic data are shown in Table 1. Both groups were similar except for the higher GC consumption and the higher lumbar bone mass of the CA group. Of the patients with CA, 41 patients received a synthetic DMARD (MTXmainly, 90\%), and 40 patients $(53.9 \%)$ received GC The average dose of DNS injections was 4.5 (1-10), with average treatment duration (range and DS) of 23.03 months $(6-66,13.3)$.The incidence of infections was $74(39.3 \%), 31$ patients had repeated infections. The most frequent were urinary tract infections (UTI), respiratory, mouth and other infections. There were 4 serious infections, 2 UTI and 2 pneumonias (both concluded in death, in patients with RA). The incidence of infections among patients with $\mathrm{OP}$ and with $\mathrm{CA}$ was similar in both groups $(40(35.7 \%)$ vs. $34(44.7 \%), p<0.209)$. Multiple regression including age, synthetic DMARD, GC and duration of treatment with DNS showed that the duration of treatment with DNS $[\operatorname{Exp}(B=1.058, p<0.001]$ and $G C$ $[\operatorname{Exp}(B)=2.484, p=0.010]$ were the only predictors of increased risk of infections.

Abstract AB1441-HPR - Table 1

\begin{tabular}{lccc}
\hline & OP $(\mathrm{n}=112)$ & $\mathrm{CA}(\mathrm{n}=76)$ & $\mathrm{p}$ \\
\hline Women, $\mathrm{n}(\%)$ & $99(88.3)$ & $66(86)$ & 0.822 \\
Age, mean (SD) & 66.9 & 67.7 & 0.622 \\
& $(10.27)$ & $(11.11)$ & \\
BMD LS, mean (SD) & $-2.9(0.98)$ & $-2.3(1.5)$ & $\mathbf{0 . 0 0 3}$ \\
EPOC, $\mathrm{n}(\%)$ & $12(10.7)$ & $11(14.4)$ & 0.499 \\
Smoking ever, $\mathrm{n}(\%)$ & $23(20.5)$ & $10(13.1)$ & 0.242 \\
Diabetes mellitus, $\mathrm{n}(\%)$ & $13(11.6)$ & $14(18.4)$ & 0.209 \\
Infections $\mathrm{n}(\%)$ & $40(35.7)$ & $34(44.7)$ & 0.227 \\
Corticosteroid dose, mean & $0.79(4.1)$ & $4(5.59)$ & $<\mathbf{0 . 0 0 0 1}$ \\
(SD) & & & \\
\hline
\end{tabular}

Conclusions: Patients with CA and OP who receive DNS and TB have a similar incidence of infections. However, the risk of infection is higher in CA patients related with the use of GC. The most frequent infection was UTI followed by respiratory infections, but they did not suppose the suspension of the treatment in the majority of the cases.

Disclosure of Interest: None declared

DOI: 10.1136/annrheumdis-2018-eular.6428

\section{AB1442-HPR A MISUNDERSTOOD BURDEN - LIVING WITH SLE IN SOUTH AFRICA}

A Phuti ${ }^{1}$, M. Schneider ${ }^{2}$, M. Tikly ${ }^{3}$, B. Hodkinson ${ }^{1} .{ }^{1}$ Medicine; ${ }^{2}$ Psychiatry and Mental Health, University of Cape Town, Cape Town; ${ }^{3}$ Medicine, University of Witwatersrand, Johannesburg, South Africa

Background: Systemic lupus erythematosus (SLE) has a profound impact on health related quality of life (HRQOL). There has been no qualitative research to the explore patients' perspectives and unmet needs in SLE in sub-Saharan Africa.

Objectives: To examine the experiences and perceptions of SLE women. Methods: In-depth interviews of 25 South African women with SLE were conducted to explore a range of $\mathrm{HRQOL}$ experiences including pain, fatigue, emotional health, sexual well-being, fertility and aesthetic concerns

Results: Most patients (72\%) were black Africans, the remaining were of mixed race ancestry, and only a quarter were employed. Living with pain was the commonest complaint, negatively impacting on activities of daily living, coping with family expectations, social life, sleep and intimacy with partners. Patients struggled to explain the pain to their families, employers, community and health care providers. Seventeen participants expressed the challenge of living with fatigue, described as "emotionally draining" and as "always tired, a person who doesn't have the energy." One patient believed that witches in church had supernatural powers that took all her energy away. Many felt their fatigue was misconstrued, and that they were labelled as 'simply lazy' by health professionals and family members. This pernicious fatigue had negative consequences on many facets of daily life, including caring for dependents, holding down steady job and sexual wellbeing. All patients experienced low emotional states ranging from anger, bitterness, anxiety, confusion, and sadness, which frequently resulted in suicidal ideation. Many patients experienced difficulties with conception, complicated pregnancies and miscarriage. The 